\title{
Regioselective Fluoroalkylphosphorylation of Unactivated Alkenes via Radical-Mediated Alkoxylphosphane Rearrangement
}

\author{
Dong-Tai Xie ${ }^{\S}$ Hong-Lei Chen, ${ }^{\S}$ Dian Wei, Bang-Yi Wei, Zheng-Hu Li, Jian-Wu Zhang, Wei Yu, and \\ Bing Han*
}

State Key Laboratory of Applied Organic Chemistry (SKLAOC), College of Chemistry and Chemical Engineering, Lanzhou University, 222 South Tianshui Road, Lanzhou, 730000, People’s Republic of China.

Supporting Information Placeholder

\begin{abstract}
A regioselective radical fluoroalkylphosphorylation of unactivated alkenes has been developed by a one-pot twosteps reaction of (bis)homoallylic alcohols, organophosphine chlorides $\left(\mathrm{R}_{2} \mathrm{PCl}\right)$, and fluoroalkyl iodides $\left(\mathrm{R}_{\mathrm{F}} \mathrm{I}\right)$ under visible light irradiation. This protocol employs the radical rearrangement of the in situ formed alkoxyphosphane for the first time to regiospecific installing a phosphonyl group onto the inner carbon of terminal olefins in alkene difunctionalization via $\mathrm{C}-\mathrm{P}$ bond formation and $\mathrm{C}-\mathrm{O}$ bond homolytic cleavage. Consequently, a series of high valueadded fluoroalkylphosphorylated alkyl iodides and alcohols are easily and efficiently synthesized by subsequent iodination and hydroxylation of the generated carbon-centered radicals.
\end{abstract}

Organophosphoryl compounds have found wide applications in various fields such as medicinal molecules, ${ }^{1}$ functional materials, ${ }^{2}$ and catalysis, ${ }^{3}$ due to their picturesque physical and chemical properties. Thus, many strategies have been developed for the incorporation of phosphoryl group into organic molecules including ionic, ${ }^{4}$ radical, ${ }^{5}$ and transition metal-catalyzed $^{6}$ C-P bond formations. Among them, phosphoryl radical-mediated difunctionalization of olefins represents an extraordinary valuable and versatile toolbox to introduce both phosphoryl as well as other useful functional groups in the molecules simultaneously. ${ }^{7}$ However, the regioselectivity of such difunctionalization is always reflected in the formation of the terminal-selective phosphorylation products since phosphonyl radical as a highly active attacking species is intrinsically attached to the end side of the terminal olefins (Scheme 1Aa). In contrast, the assembly of phosphoryl onto the internal side of terminal olefins is hard to obtain. One possible tactic is to use radical replacement reaction between phosphines and the alkyl radicals derived from alkenes to realize C-P bond construction, followed by the conversion of $\mathrm{P}^{\mathrm{III}}$ to $\mathrm{P}^{\mathrm{V}}$ by treating with extra oxidants ${ }^{8}$ (Scheme $1 \mathrm{Ab}$ ). However, common phosphines such as alkyl/aryl/alkoxy/ aryloxy phosphines cannot undergo such transformation by forming corresponding phosphoranyl radicals due to their low reactivity ${ }^{9}$ (Scheme 1Ac). Thus, a few successful cases have had to use presynthesized highly reactive phosphines $\mathrm{Me}_{3} \mathrm{X}$ $\mathrm{PPh}_{2}(\mathrm{X}=\mathrm{Sn} \text { or } \mathrm{Si})^{8 \mathrm{a}-8 \mathrm{c}}$ and $\mathrm{Ph}_{2} \mathrm{P}(\mathrm{O})-\mathrm{PPh}_{2}{ }^{8 \mathrm{~d}-8 \mathrm{e}}$ to enable the substitution by delivering sufficient $\alpha$-scission driven forces.

\section{Scheme 1. Regioselective Phosphorylation of Alkenes}

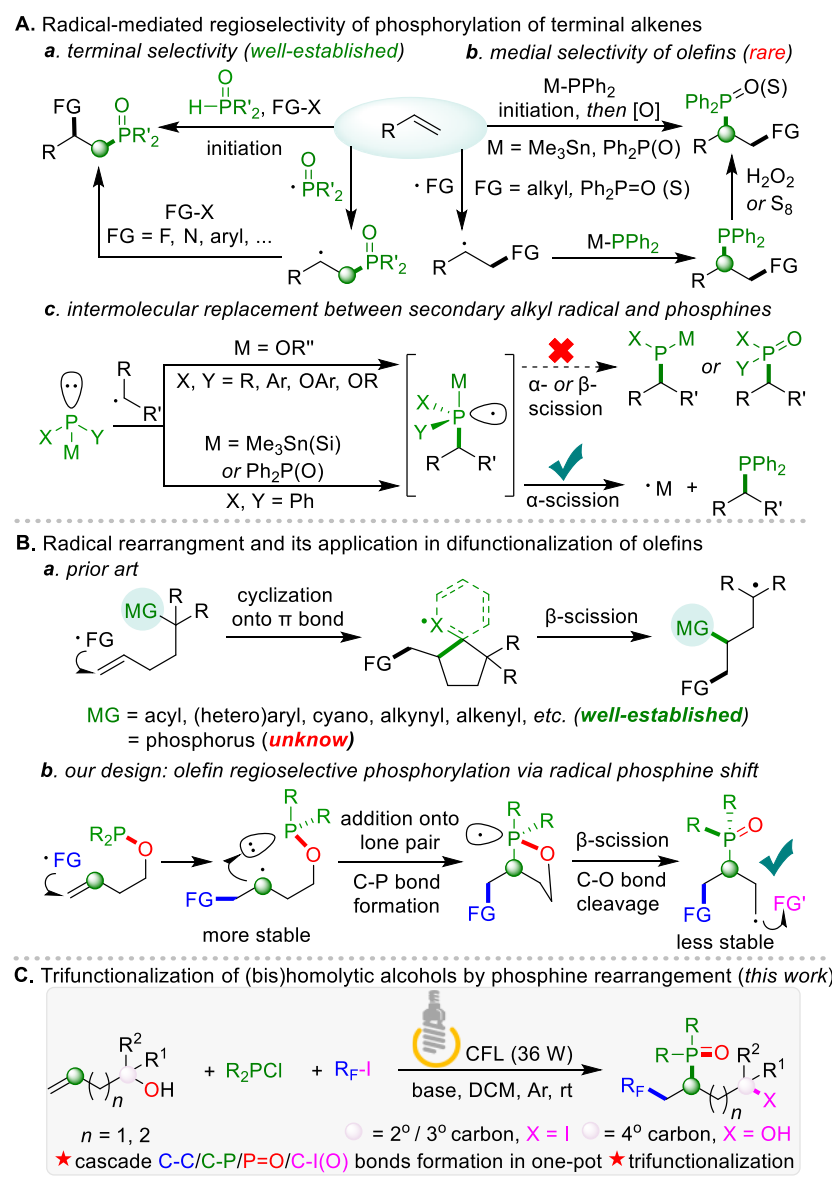

Rearrangement reaction, as one of the most significant transformations, has been widely applied to improve synthetic efficiency and molecular complexity. ${ }^{10}$ Recently, radicalmediated functional group rearrangement has proven to be an efficient and elegant mean for olefin difunctionalization. By combing the intermolecular radical addition onto olefin and subsequent radical cyclization and $\beta$-fragmentation, this protocol realizes olefin difunctionalization by migrating functional groups bearing $\pi$ bonds from the distal position of molecules to the medial carbon of terminal olefin ${ }^{11}$ (Scheme $1 \mathrm{Ba})$. Unfortunately, this strategy is rarely used for the migration of functional groups with lone pair electrons ${ }^{12}$ such 
as alkoxy phosphine. ${ }^{13}$ Despite the intermolecular substitution between alkyl radical and alkoxyphosphine has shown to be fail (Scheme 1Ac), we envisioned its intramolecular mode may be feasible and can be used as the key link to realize the regioselective phosphorylation of olefins. We hypothesized that if a radical is added onto the alkene moiety of alkoxy phosphine adduct formed in situ from homoallylic alcohol and diphenyl phosphine chloride, and the resulting secondary alkyl radical may further cyclize onto the tethered phosphine to produce phosphoranyl radical. If so, by taking advantage of the subsequent $\beta$-scission of $\mathrm{C}-\mathrm{O}$ bond and further functionalization of the formed primary carbon-centered radical, it is possible to achieve the regioselective introduction of phosphoryl at the internal side of terminal alkenes without utilizing special phosphines and extra oxidants; meanwhile, the dehydroxylative trifunctionalization of homoallylic alcohols can be also realized (Scheme 1Bb). Although such rearrangement involves the generation of unstable primary carbon radical and the homolysis of strong $\mathrm{C}-\mathrm{O}$ bond, the generation of $\mathrm{C}-\mathrm{P} \sigma$ bond and $\mathrm{P}=\mathrm{O} \pi$ bond may compensate for this endothermic enthalpy and facilitate the reaction. Herein, we demonstrate realization of this goal in the case of regioselective fluoroalkyl phosphorylation of unactivated olefines by a one-pot multi-component reaction of commercially available (bis)homoallylic alcohols, $\mathrm{R}_{2} \mathrm{PCl}$, and fluoroalkyl iodides under visible light irradiation (Scheme 1C). Consequently, a series of vicinal fluoroalkylphosphorylated

Table 1. Optimization of the Reaction Conditions ${ }^{a}$

\begin{tabular}{|c|c|c|c|}
\hline$\overbrace{\text { a1 (1.0 equiv.) }}^{\mathrm{OH}}$ & 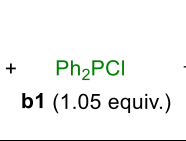 & $\begin{array}{c}\text { base }(1.0 \text { equiv) } \\
\text { solvent }(0.1 \mathrm{M}), \mathrm{Ar}, \mathrm{rt}, 1 \mathrm{~h} \\
\underset{\text { then } n \mathrm{C}_{4} \mathrm{~F}_{9^{-I}}(\mathrm{c} 1,2.0 \text { equiv. })}{\longrightarrow} \\
\mathrm{Ar}, \mathrm{rt}, \mathrm{CFL}(36 \mathrm{~W}), 5 \mathrm{~h}\end{array}$ & $\underbrace{\mathrm{Ph}}_{\mathbf{d} \mathbf{1}}$ \\
\hline entry & base & solvent & yield $(\%)^{b}$ \\
\hline 1 & $\mathrm{NEt}_{3}$ & DCM & 70 \\
\hline 2 & - & DCM & 0 \\
\hline 3 & HNEt $_{2}$ & DCM & 75 \\
\hline 4 & $n \mathrm{Bu}_{2} \mathrm{NH}$ & DCM & 61 \\
\hline 5 & DABCO & DCM & 38 \\
\hline 6 & DBU & DCM & 64 \\
\hline 7 & TMEDA & DCM & 44 \\
\hline 8 & DIPEA & DCM & 8 \\
\hline 9 & $\mathrm{NaOAc}$ & DCM & 29 \\
\hline 10 & $\mathrm{~K}_{3} \mathrm{PO}_{4}$ & DCM & 5 \\
\hline 11 & $\mathrm{HNEt}_{2}$ & DCE & 73 \\
\hline 12 & $\mathrm{HNEt}_{2}$ & $\mathrm{CH}_{3} \mathrm{CN}$ & 53 \\
\hline 13 & $\mathrm{HNEt}_{2}$ & THF & 21 \\
\hline 14 & $\mathrm{HNEt}_{2}$ & $\mathrm{PhMe}$ & 12 \\
\hline 15 & $\mathrm{HNEt}_{2}$ & $\mathrm{DMF}$ & 0 \\
\hline $16^{c}$ & $\mathrm{HNEt}_{2}$ & DCM & 25 \\
\hline $17^{d}$ & $\mathrm{HNEt}_{2}$ & DCM & 68 \\
\hline
\end{tabular}

${ }^{a}$ Reaction was conducted on 0.2 mmol scale. ${ }^{b}$ Isolated yield. ${ }^{c}$ Without light irradiation. ${ }^{d}$ Blue LEDs (460 nm, $\left.24 \mathrm{~W}\right)$ was used instead of CFL. DABCO = triethylenediamine, DBU $=1,8$-diazabicyclo[5.4.0] undec-7-ene, TMEDA $=N, N, N$ ', $N$ '-tetramethylethylenediamine, DIPEA $=\mathrm{N}, \mathrm{N}$-diisopropylethylamine, $\mathrm{DCM}=$ dichloromethane, $\mathrm{DCE}=$ dichloroethane, $\mathrm{THF}=$ tetrahydrofuran, $\mathrm{DMF}=\mathrm{N}, \mathrm{N}$-dimethylformamide. alkyl iodides and alcohols are conveniently synthesized. As the introduction of fluorine into bioactive molecules can significantly enhance their metabolic stability, solubility, permeability, and lipophilicity, ${ }^{14}$ incorporating fluorine or fluorine-containing groups into organic phosphonyls are exceptionally meaningful. ${ }^{15}$ In this context, our study represents the first example for the synthesis of such compounds by the regioselective incorporation of fluoroalkyl and phosphoryl simultaneously into olefins.

Our studies were commenced with a one-pot twosteps model reaction of homoallylic alcohol (a1), $\mathrm{Ph}_{2} \mathrm{PCl}(\mathbf{b 1})$, base, and $n \mathrm{C}_{4} \mathrm{~F}_{9} \mathrm{I}$ (c1) in DCM (dichloromethane) under argon atmosphere at room temperature, followed by the irradiation with CFL (compact fluorescence light, $36 \mathrm{~W}$ ) as shown in Table 1. Delightedly, the desired phosphorous rearrangement took place smoothly and gave the regioselective trifunctionalized product fluoroalkylphosphorylated alkyl iodide $\mathbf{d 1}$ in $70 \%$ yield when triethylamine was used as base; whereas no product d1 was produced under base-free conditions (Table 1, entries 1 and 2). The yield of d1 increased to $75 \%$ when $\mathrm{HNEt}_{2}$ was employed instead of triethylamine, (Table 1, entry 3). Other organic and inorganic bases such as $n \mathrm{Bu}_{2} \mathrm{NH}$, DABCO, DBU, TMEDA, DIPEA, NaOAc, and $\mathrm{K}_{3} \mathrm{PO}_{4}$ could also promote this conversion but gave d1 in unsatisfactory yields (Table 1, entries 4-10). The results of solvents investigation for $\mathrm{CH}_{3} \mathrm{CN}$, THF, toluene, and DMF showed that no better yield was obtained (Table 1, entries 1215). Light irradiation was essential for an efficient reaction since d1 was only formed in $25 \%$ yield without light; replacing the light source from CFL to blue LEDs (lightemitting diodes, $460 \mathrm{~nm}, 24 \mathrm{~W}$ ) led to a slightly lower yield (Table 1, entries 16 and 17).

With the optimum reaction conditions established (entry 2 in Table 1), we set about to evaluate the generality of this method, and the results are illustrated in Scheme 2. The scope of $\mathrm{R}_{2} \mathrm{PCl}$ was firstly explored (Scheme 2A). Diphenylchlorophosphines bearing a variety of substituents with different electronical properties such as $\mathrm{MeO}, \mathrm{Me}, \mathrm{Cl}$, and $\mathrm{F}$ on phenyl ring at para-position participated very well in the reaction, providing the desired products $\mathbf{d 2}$-d5 in good yields. The structure of trifunctionalized product $\mathbf{d} \mathbf{4}$ was further confirmed by X-ray crystallographic analysis. 2-Methyl and 3,5-dimethyl substituted $\mathrm{Ar}_{2} \mathrm{PCl}$ were also performed well, giving rise to the products $\mathbf{d 6}$ and $\mathbf{d} 7$ in $40 \%$ and $56 \%$ yields, respectively. In addition, the reaction was compatible with dinaphthyl chlorophosphines, as demonstrated in the case of d8. Notably, besides $\mathrm{Ar}_{2} \mathrm{PCl}$, dialkyl and dialkoxy substituted chlorophosphines were also good candidates for this transformation, furnishing the desired products d9-d11, albeit in slightly lower yields. Next, a variety of fluoroalkyl iodides were examined as depicted in Scheme 2B. A wide array of linear and branched perfluoroalkyl iodides with different length reacted smoothly in this tactic, giving products d12-d17 in excellent yields. Other hybrid fluoroalkyl iodides involving $\mathrm{Cl}, \mathrm{Br}$, I, ester, sulfonyl fluoride, and sulfamide were all suitable to the conversion by chemoselective cleavage of C-I bond to give the corresponding products d18-d23. After that, we examined the scope of the homoallylic alcohols as illustrated in Scheme 3B. Primary homoallylic alcohols bearing methyl, ethyl, and benzyl substituents on the terminal alkene moiety were all compatible with this way, affording the corresponding $\alpha$ tertiary phosphoryl products $\mathbf{d 2 4 - \mathbf { d } 2 6}$ in excellent yields. When inner alkenes such as cyclopentenyl and cyclohexenyl 


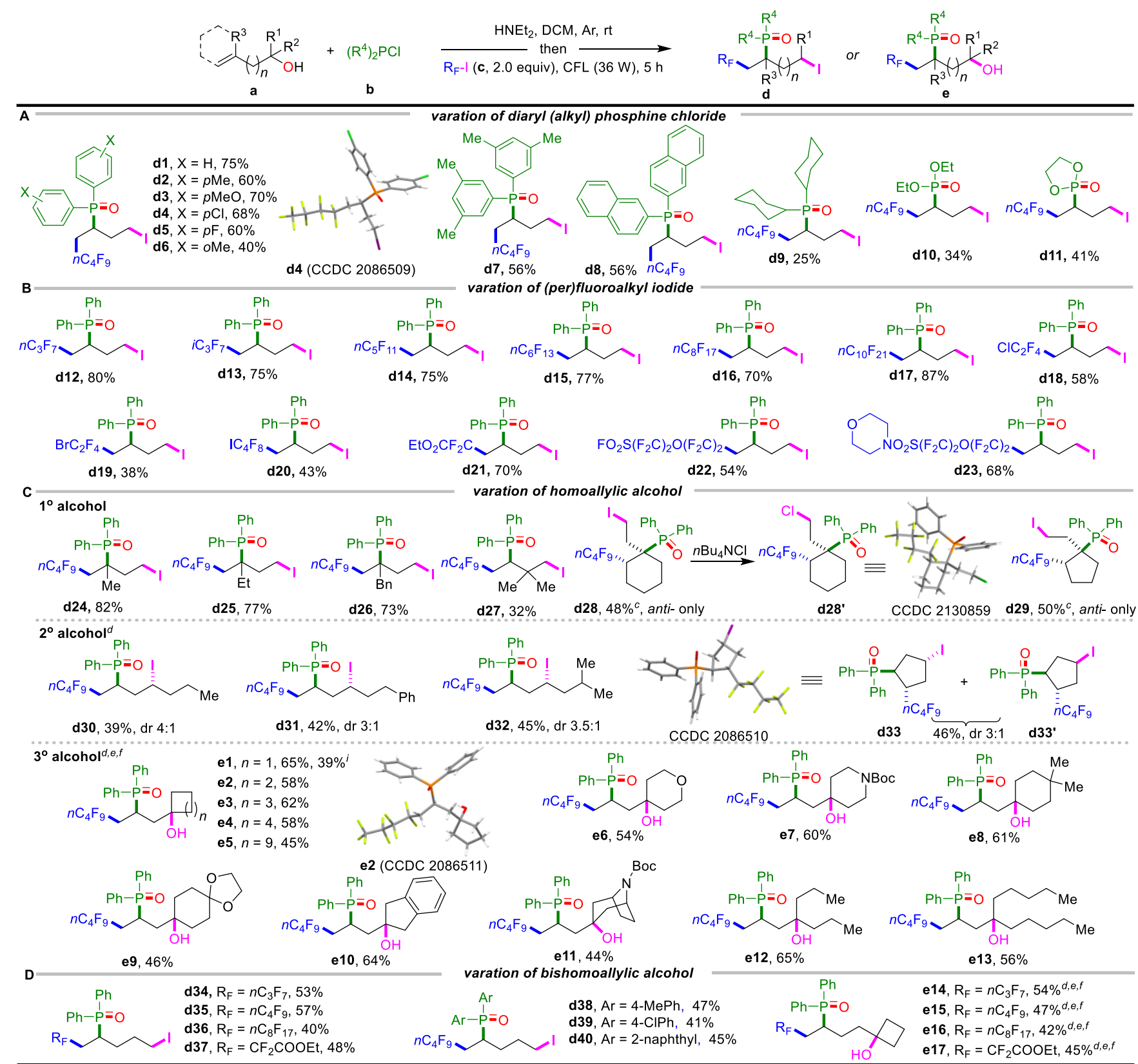

${ }^{a} \mathrm{~A}$ mixture of a $\left(0.2 \mathrm{mmol}, 1.0\right.$ equiv.), b (1.05 equiv.), and $\mathrm{HNEt}_{2}$ (1.0 equiv.) in DCM (2 mL) was stirred at $\mathrm{rt}$ under $\mathrm{Ar}$ till the substitution was complete. Then $\mathrm{R}_{\mathrm{F}} \mathrm{I}$ (2.0 equiv.) was added and the mixture was stirred for additional $5 \mathrm{~h}$ under CFL irradiation (36 W) at rt under Ar. ${ }^{b}$ Isolated yields. ${ }^{c}$ For optimal conversion, the adduct of $\mathbf{a}$ and $\mathbf{b}$ was first isolated and then being subjected to the photoreaction conditions. ${ }^{d}$ Dimethylaminopyridine $(0.06 \mathrm{mmol}, 30 \mathrm{~mol} \%)$ was added to promote substitution reaction. ${ }^{e} \mathrm{NEt}_{3}(1.5$ equiv.) was used instead of $\mathrm{HNEt}_{2} .{ }^{f} \mathrm{H}_{2} \mathrm{O}$ (1.0 equiv.) was added along with $\mathrm{R}_{\mathrm{F}}-\mathrm{I} .{ }^{i}$ Without extra $\mathrm{H}_{2} \mathrm{O}$.

were merged in primary homoallylic alcohols, the reaction also converted well to provide regio- and stereospecific antifluoroalkylphosphorylation products $\mathbf{d 2 8}$ and $\mathbf{~} \mathbf{2 9}$ in moderate yields. The anti-configuration was confirmed by X-ray crystallographic analysis of its derivative d28' of $\mathbf{d 2 8}$. Terminal alkene incorporated secondary homoallylic alcohols with different structures could also be applied to the agreement and gave products $\mathbf{d 3 0 - d 3 2}$ in moderate yields with 4:1-3:1 diastereoselectivity. Significantly, when cyclic secondary homoallylic alcohols like cyclopent-3-en-1-ol was involved in the reaction, trifunctionalized cyclopentanes $\mathbf{d 3 3}$ and $\mathbf{~ d 3 3}$ ' were generated as the epimers in a combined yield of
$46 \%$ with 3:1 stereoselectivity. The structure of major epimer d33 was determined by X-ray crystallographic analysis. Notably, tertiary homoallylic alcohols were also compatible with this regioselective fluoroalkylphosphorylation by affording products in form of aliphatic alcohols rather than alkyl iodides. Apparently, the newly introduced hydroxyl group was derived from the trace amount of water in extra dry DCM. Indeed, the addition of 1.0 equivalent water could further increase the yields of products. As a result, a variety of cyclic tertiary homoallylic alcohols with different cycloalkyl ring sizes were transformed to the fluoroalkylphosphorylated alcohols e1-e5 in good yields. The alcohol structure was also 
confirmed by X-ray crystallographic analysis of $\mathbf{e 2}$. Heteroatoms such as $\mathrm{O}$ - / $\mathrm{N}$-atom embedded and gem-dimethyl substituted homoallylic cycloalcohols were converted to the corresponding products e6-e8 in high yields. In addition, the conversion of spiro, fused, and bridged homoallylic cycloalcohols to the desired products e9-e11 was also successful. On the other hand, tertiary alcohols of open chain could also react efficiently under our system, as demonstrated in the cases of $\mathbf{e 1 2}$ and $\mathbf{e 1 3}$. It is noteworthy that this approach worked very well for bishomoallylic alcohols too, yielding the corresponding trifunctionalized products d34-d40 and e14-e17 through a radical 6-membered ring phosphorous intermediate rearrangement.

The synthetic practicality of this strategy and the versatility of products are proved as shown in Scheme 3. A gram-scale synthesis of d1 $(1.43 \mathrm{~g}, 68 \%$ yield $)$ was successfully proceeded on $3.5 \mathrm{mmol}$ scale. The follow-up derivatization of d1 was performed by the nucleophilic substitution, reduction, and arylation of C-I bond, affording the corresponding derivatives $\mathbf{f}-\mathbf{l}$ in good yields.

\section{Scheme 3. Practicality and Follow-up Transformations}

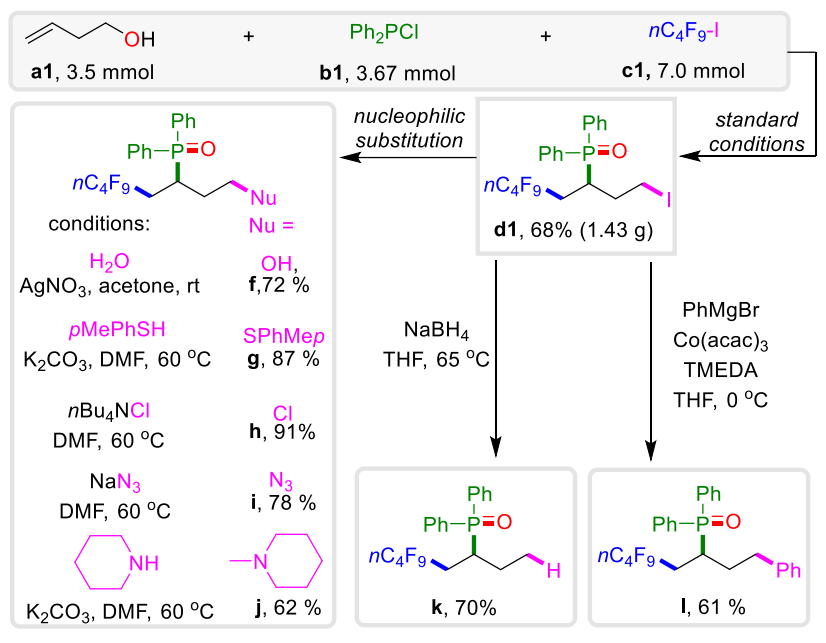

To account for the mechanism of this reaction, a series of control experiments were carried out as depicted in Scheme 4A. The reaction of $\mathbf{a} 1$ with $\mathbf{b 1}$ under the conditions of $\mathrm{NHEt}_{2}$ gave the adduct I-1 in nearly quantitative yield (Scheme 4Aa). When I-1 was used as the substrate to react with $n \mathrm{C}_{4} \mathrm{~F}_{9} \mathrm{I}$ without $\mathrm{HNEt}_{2}$, the reaction took place very well under visible light irradiation and gave d1 in $78 \%$ yield (Scheme $4 \mathrm{Ab}$ ). These results not only confirm that $\mathrm{HNEt}_{2}$ serves as base to promote the reaction of homoallylic alcohols and phosphines to form adduct I-1 but also reveals that $\mathbf{I - 1}$ is the key intermediate to react with $n \mathrm{C}_{4} \mathrm{~F}_{9} \mathrm{I}$ to take place phosphorous rearrangement-mediated trifuntionalization. When cyclopropyl incorporated adduct $\mathbf{I - 2}$ was employed to react with $n \mathrm{C}_{4} \mathrm{~F}_{9} \mathrm{I}$, the cyclopropyl ring-opening product $\mathbf{d 4 1}$ was obtained in $55 \%$ yield (Scheme 4Ac). This "radical clock" experiment ${ }^{16}$ clearly indicates that a radical-mediated phosphorous rearrangement process is involved in the reaction. In addition, isotope-labeling experiment by utilizing $\mathrm{H}_{2}{ }^{18} \mathrm{O}$ clearly verifies that the newly introduced hydroxyl in products e came from Scheme 4. Mechanistic Studies and Proposed Mechanism
A) Control Experiments

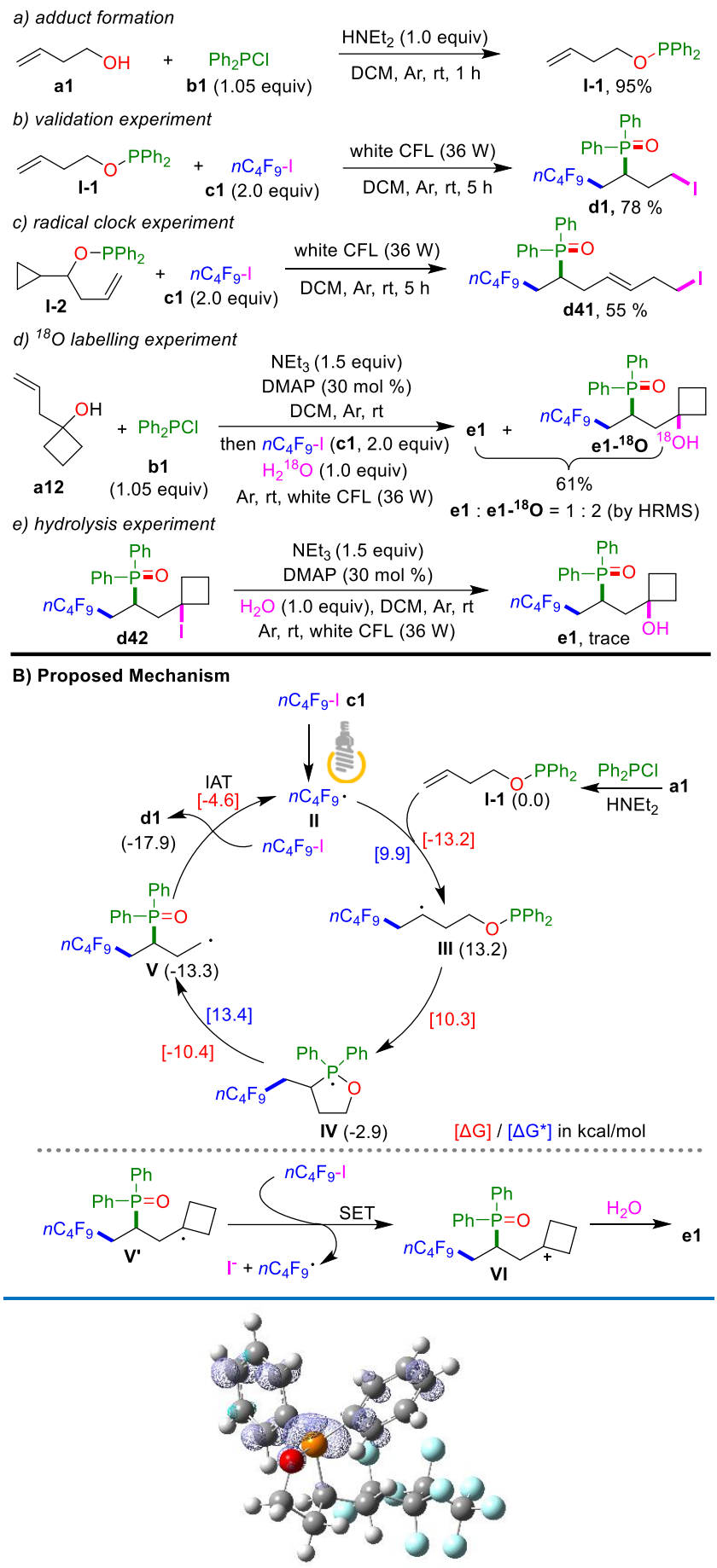

Figure 1. Spin density (B3LYP/6-311+G (d,p), isosurface value $=0.006)$ of radical $\mathbf{I V}$.

water (Scheme 4Ad). However, e does not come from alkaline hydrolysis of its corresponding tertiary alkyl iodide $\mathbf{d}$ since almost no hydrolysis occurred when $\mathbf{d}$ was treated under the standard base conditions. Thus, the production of $\mathbf{e}$ can be attributed to the fact that tertiary radicals derived from tertiary alcohols are easily oxidized by fluoroalkyl iodides to form carbocations, which incline to react with water to produce alcohols rather than iodide anion to yield aliphatic iodides.

On the basis of our experimental observation, a proposed mechanism is delineated by a representative sample in Scheme 
4B. A base-promoted nucleophilic substitution of $\mathrm{Ph}_{2} \mathrm{PCl}$ b1 and homoallylic alcohol a1 occurs first to provide the adduct I-1. Then $\mathrm{C}_{4} \mathrm{~F}_{9}$ radical II which is initiated by the irradiation of $\mathrm{C}_{4} \mathrm{~F}_{9}$-I under visible light, ${ }^{17}$ adds onto the alkene moiety of adduct I-1 to yield the C-centered radical intermediate III through a $9.9 \mathrm{kcal} / \mathrm{mol}$ reaction energy barrier (see the Supporting Information for the details of DFT (density functional theory) calculations). Despite it is not a main process in our reaction, the generation of $\mathrm{C}_{4} \mathrm{~F}_{9}$ radical can also be obtained from an EDA (electron donor-acceptor) complex of the electron-rich phosphine adduct $\mathbf{I}-\mathbf{1}$ and electrondeficient $\mathrm{C}_{4} \mathrm{~F}_{9} \mathrm{I}^{18}{ }^{18}$ III experiences a fast radical cyclization onto the lone electron pairs of phosphine to yield phosphoranyl radical intermediate $\mathbf{I V}$, which subsequently undergoes $\mathrm{C}-\mathrm{O}$ bond $\beta$-fragmentation across an energy barrier of 13.4 $\mathrm{kcal} / \mathrm{mol}$ to form the $\mathrm{C}$-radical intermediate $\mathbf{V}$. The calculated Gibbs free energy change of this radical phosphine rearrangement is only $-0.1 \mathrm{kcal} / \mathrm{mol}$, which is almost enthalpy neutral. Finally, product $\mathbf{d} \mathbf{1}$ is formed by the reaction of $\mathbf{V}$ and $\mathrm{C}_{4} \mathrm{~F}_{9} \mathrm{I}$ via an iodine-atom transfer (IAT) process. When the reaction involves tertiary alcohol, as in the case of $\mathbf{e 1}$, the formed tertiary radical $\mathbf{V}^{\prime}$ tends to undergo a single-electron transfer (SET) process rather than an IAT process to react with $\mathrm{C}_{4} \mathrm{~F}_{9} \mathrm{I},{ }^{18 a, 19}$ providing carbocation $\mathrm{VI}, \mathrm{C}_{4} \mathrm{~F}_{9}$ radical, and iodide anion. The hydroxylation of $\mathbf{V I}$ by water affords $\mathbf{e}$.

The calculated spin density map of intermediate IV clearly indicates that the spin is delocalized on P-atom (0.68) and the tethered two phenyl rings (0.33) (Figure 1), manifesting the formation of phosphoranyl radical. EPR (electronic paramagnetic resonance) experiments also detected the signals of PBN (phenyl $N$-tert-butylnitrone) trapped $\mathrm{P}^{\mathrm{V}}$-centered radical $^{20}$ IV and $\mathrm{C}_{4} \mathrm{~F}_{9}$ radical $\mathbf{I I}$, respectively, which further confirmed that this reaction involves a radical-mediated phosphine rearrangement process (see the Supporting Information for the details of EPR experiments).

In summary, we have successfully developed a novel, facile, and efficient approach for the regioselective fluoroalkyl phosphorylation of unactivated alkenes by a one-pot twosteps reaction of readily accessible (bis)homoallylic alcohols, organophosphine chlorides, and fluoroalkyl iodides under visible light irradiation. The protocol employs radical phosphine rearrangement as the key step to realize the unusual installing phosphoryl in the inner side of the terminal olefins by using commercially available common organic chlorophosphines without extra oxidants. This tactic not only provides remarkable opportunities for the regioselective introducing fluoroalkyl and phosphoryl simultaneously in olefins and the trifunctionalizing (bis)homoallylic alcohols, but also broadens new boundaries of radical rearrangement modes of phosphines and their synthetic application. The further exploration of such radical rearrangement for synthetic purposes are ongoing in our laboratory.

\section{ASSOCIATED CONTENT}

\section{Supporting Information}

Experimental procedures, spectral characterization, crystallographic data, and DFT calculations (PDF).

\section{AUTHOR INFORMATION}

\section{Corresponding Author}

Bing Han - State Key Laboratory of Applied Organic Chemistry, College of Chemistry and Chemical Engineering, Lanzhou
University, Lanzhou 730000, China; Email: hanb@1zu.edu.cn, ORCID: 0000-0003-0507-9742

\section{Authors}

Dong-Tai Xie - State Key Laboratory of Applied Organic Chemistry, College of Chemistry and Chemical Engineering, Lanzhou University, Lanzhou 730000, China

Hong-Lei Chen - State Key Laboratory of Applied Organic Chemistry, College of Chemistry and Chemical Engineering, Lanzhou University, Lanzhou 730000, China

Dian Wei - State Key Laboratory of Applied Organic Chemistry, College of Chemistry and Chemical Engineering, Lanzhou University, Lanzhou 730000, China

Bang-Yi Wei - State Key Laboratory of Applied Organic Chemistry, College of Chemistry and Chemical Engineering, Lanzhou University, Lanzhou 730000, China

Zheng-Hu Li - State Key Laboratory of Applied Organic Chemistry, College of Chemistry and Chemical Engineering, Lanzhou University, Lanzhou 730000, China

Jian-Wu Zhang - State Key Laboratory of Applied Organic Chemistry, College of Chemistry and Chemical Engineering, Lanzhou University, Lanzhou 730000, China

Wei Yu - State Key Laboratory of Applied Organic Chemistry, College of Chemistry and Chemical Engineering, Lanzhou University, Lanzhou 730000, China

\section{Author Contributions}

§These authors contributed equally to this work.

\section{Notes}

The authors declare no competing financial interests.

\section{ACKNOWLEDGMENTS}

We thank the National Natural Science Foundation of China (Nos. 21873041, 22171118, and 21632001) and the "111" Project for financial support.

\section{REFERENCES}

(1) (a) Pradere, U.; Garnier-Amblard, E. C.; Coats, S. J.; Amblard, F.; Schinazi, R. F. Synthesis of Nucleoside Phosphate and Phosphonate Prodrugs. Chem. Rev. 2014, 114, 9154-9218. (b) Combs, A. P. Recent Advances in the Discovery of Competitive Protein Tyrosine Phosphatase 1B Inhibitors for the Treatment of Diabetes, Obesity, and Cancer. J. Med. Chem. 2010, 53, 2333-2344.

(2) (a) Queffélec, C.; Petit, M.; Janvier, P.; Knight, D. A.; Bujoli, B. Surface Modification Using Phosphonic Acids and Esters. Chem. Rev. 2012, 112, 3777-3807. (b) Baumgartner, T.; Re'au, R. Organophosphorus $\pi$-Conjugated Materials. Chem. Rev. 2006, 106, 4681-4727. (3) Onouchi, H.; Miyagawa, T.; Furuko, A.; Maeda, K.; Yashima, E. Enantioselective Esterification of Prochiral Phosphonate Pendants of a Polyphenylacetylene Assisted by Macromolecular Helicity: Storage of a Dynamic Macromolecular Helicity Memory. $J$. Am. Chem. Soc. 2005, 127, 2960-2965.

(3) Xie, C. M.; Smaligo, A. J.; Song, X.-R.; Kwon, O. PhosphorusBased Catalysis. ACS Cent. Sci. 2021, 7, 536-558.

(4) For selected reviews, see: (a) Bhattacharya, A. K.; Thyagarajan, G. Michaelis-Arbuzov Rearrangement. Chem. Rev. 1981, 81, 415-430 (b) Redmore, D. Heterocyclic Systems Bearing Phosphorus Substituents. Synthesis and Chemistry. Chem. Rev. 1971, 71, 315-337.

(5) For selected works, see: (a) Liu, Y.; Chen, X.-L.; Li, X.-Y.; Zhu, S.-S.; Li, S.-J.; Song, Y.; Qu, L.-B.; Yu, B. 4CzIPN-'Bu-Catalyzed Proton-Coupled Electron Transfer for Photosynthesis of Phosphorylated $N$-Heteroaromatics. J. Am. Chem. Soc. 2021, 143, 964-972. (b) Chen, Y.-R.; Duan, W.-L. Silver-Mediated Oxidative C-H/P-H Functionalization: An Efficient Route for the Synthesis of Benzo[b]phosphole Oxides. J. Am. Chem. Soc. 2013, 135, 16754 16757. (c) Jin, S. J.; Haug, G. C.; Nguyen, V. T.; Flores-Hansen, C.; 
Arman, H. D.; Larionov, O. V. Decarboxylative Phosphine Synthesis: Insights into the Catalytic, ACS Catal. 2019, 9, 9764-9774.

Autocatalytic, and Inhibitory Roles of Additives and Intermediates.

(6) For selected review and works, see: (a) Schwan, A. L. Palladium catalyzed cross-coupling reactions for phosphorus-carbon bond formation. Chem. Soc. Rev. 2004, 33, 218-224. (b) Hirai, T.; Han, L.B. Palladium-Catalyzed Insertion of Isocyanides into $\mathrm{P}(\mathrm{O})-\mathrm{H}$ Bonds: Selective Formation of Phosphinoyl Imines and Bisphosphinoylaminomethanes. J. Am. Chem. Soc. 2006, 128, 74227423. (c) Feng, C.-G.; Ye, M. C.; Xiao, K.-J.; Li, S. H; Yu, J.-Q Pd(II)-Catalyzed Phosphorylation of Aryl C-H Bonds. J. Am. Chem. Soc. 2013, 135, 9322-9325. (d) Liu, C. W.; Szostak, M. Decarbonylative Phosphorylation of Amides by Palladium and Nickel Catalysis: The Hirao Cross-Coupling of Amide Derivatives. Angew. Chem., Int. Ed. 2017, 56, 12718-12722.

(7) For selected works, see: (a) Fu, N. K.; Song, L.; Liu, J. J.; Shen, Y. F.; Siu, J. C.; Lin, S. New Bisoxazoline Ligands Enable Enantioselective Electrocatalytic Cyanofunctionalization of Vinylarenes. J. Am. Chem. Soc. 2019, 141, 14480-14485. (b) Li, Y.M.; Sun, M.; Wang, H.-L.; Tian, Q.-P.; Yang, S.-D. Direct Annulations toward Phosphorylated Oxindoles: Silver-Catalyzed Carbon-Phosphorus Functionalization of Alkenes. Angew. Chem., Int. Ed. 2013, 52, 3972-3976. (c) Wei, W.; Ji, J.-X. Catalytic and Direct Oxyphosphorylation of Alkenes with Dioxygen and H-Phosphonates Leading to $\beta$-Ketophosphonates. Angew. Chem., Int. Ed. 2011, 50 , 9097-9099. (d) Fu, Q.; Bo, Z.-Y; Ye, J.-H.; Ju, T.; Huang, H.; Liao L.-L.; Yu, D.-G. Transition Metal-free Phosphonocarboxylation of Alkenes with Carbon Dioxide via Visible-light Photoredox Catalysis. Nat. Commun. 2019, 10, 3592.

(8) (a) Lamas, M. C.; Studer, A. Radical Alkylphosphanylation of Olefins with Stannylated or Silylated Phosphanes and Alkyl Iodides. Org. Lett. 2011, 13, 2236-2239. (b) Otomura, N.; Okugawa, Y.; Hirano, K.; Miura, M. vic-Diphosphination of Alkenes with Silylphosphine under Visible-Light-Promoted Photoredox Catalysis. Org. Lett. 2017, 19, 4802-4805. (c) Okugawa, Y.; Hirano, K.; Miura, M. Copper-Catalyzed Vicinal Diphosphination of Styrenes: Access to 1,2-Bis(diphenylphosphino)ethane-Type Bidentate Ligands from Olefins. Angew. Chem. Int. Ed. 2016, 55, 13558-13561. (d) Sato, Y.; Kawaguchi, S.-i.; Nomoto, A.; Ogawa, A. Highly Selective Phosphinylphosphination of Alkenes with Tetraphenyldiphosphine Monoxide. Angew. Chem. Int. Ed. 2016, 55, 9700-9703. (e) Sato, Y.; Kawaguchi, S.-I.; Nomoto, A.; Ogawa, A. Chem. Eur. J. 2019, 25, 2295-2302.

(9) (a) Bentrude, W. G. Phosphoranyl radicals-their structure, formation, and reactions. Acc. Chem. Res. 1982, 15, 117-125. (b) Bentrude, W. G. The Chemistry of Organophosphorus Compounds; Hartley, F. R., Ed.; Wiley: Chichester, 1990; Vol. 1, pp 531-566. (c) Marque, S.; Tordo, P. Reactivity of Phosphorus Centered Radicals. Top. Curr. Chem. 2005, 250, 43-76. (d) James A. Rossi-Ashton, J. A.; Clarke, A. K.; William P. Unsworth, W. P.; Taylor, R. J. K. Phosphoranyl Radical Fragmentation Reactions Driven by Photoredox Catalysis. ACS Catal. 2020, 10, 7250-7261.

(10) For selected recent reviews, see: (a) Korb, M.; Lang, H. The anionic Fries rearrangement: a convenient route to orthofunctionalized aromatics. Chem. Soc. Rev. 2019, 48, 2829-2882. (b) Dong, S. X.; Liu, X. H.; Feng, X. M. Asymmetric Catalytic Rearrangements with $\alpha$-Diazocarbonyl Compounds. Acc. Chem. Res. 2022, 55, 415-428. (c) Wu, X. X.; Ma, Z. G.; Feng, T. T.; Zhu, C. Radical-mediated rearrangements: past, present, and future. Chem. Soc. Rev. 2021, 50, 11577-11613. (d) Li, W. P.; Xu, W. T.; Xie, J.; Yu, S. Y.; Zhu, C. J. Distal radical migration strategy: an emerging synthetic means. Chem. Soc. Rev. 2018, 47, 654-667. (e) Wu, X.-X.; Zhu, C. Radical-Mediated Remote Functional Group Migration. Acc. Chem. Res. 2020, 53, 1620-1636. (f) Chen, Y.-J.; Qu, Y.-L.; Li, X.; Wang, C.-C. Recent advances in 1,4-functional group migrationmediated radical fluoroalkylation of alkenes and alkynes. Org. Biomol. Chem. 2020, 18, 8975-8993.

(11) For selected works, see: (a) Li, Z.-L.; Li, X-H.; Wang, N.; Yang, N.-Y.; Liu, X.-Y. Radical-Mediated 1,2-Formyl/Carbonyl Functionalization of Alkenes and Application to the Construction of Medium-Sized Rings. Angew. Chem., Int. Ed. 2016, 55, 15100-
15104. (b) Monos, T. M.; McAtee, R. C.; Stephenson, C. R. J. Arylsulfonylacetamides as bifunctional reagents for alkene aminoarylation. Science. 2018, 361, 1369-1373. (c) Wu, Z.; Wang, D. P.; Liu, Y.; Huan, L. T.; Zhu, C. Chemo- and Regioselective Distal Heteroaryl ipso-Migration: A General Protocol for Heteroarylation of Unactivated Alkenes. J. Am. Chem. Soc. 2017, 139, 1388-1391. (d) Wu, Z.; Ren, R. G.; Zhu, C. Combination of a Cyano Migration Strategy and Alkene Difunctionalization: The Elusive Selective Azidocyanation of Unactivated Olefins. Angew. Chem., Int. Ed. 2016, 55, 10821-10824. (e) Tang, X. J; Studer, A. Alkene 1,2Difunctionalization by Radical Alkenyl Migration. Angew. Chem., Int Ed. 2018, 57, 814-817. (f) Xu, Y.; Wu, Z.; Jiang, J. X.; Ke, Z. F.; Zhu, C. Merging Distal Alkynyl Migration and Photoredox Catalysis for Radical Trifluoromethylative Alkynylation of Unactivated Olefins. Angew. Chem., Int. Ed. 2017, 56, 4545-4548. (g) Wei, D.; Liu, T. M.; He, Y. H.; Wei, B. Y.; Pan, J. H.; Zhang, J. W.; Jiao, N.; Han, B. Radical 1,4/5-Amino Shift Enables Access to Fluoroalkyl-Containing Primary $\beta(\gamma)$-Aminoketones under Metal-Free Conditions. Angew. Chem. Int. Ed. 2021, 60, 26308-26313. (h) Zhu, Y. C.; Zhang, Z. Y.; Jin, R.; Liu, J. Z.; Liu, G. Q.; Han, B.; Jiao, N. DMSO-Enabled Selective Radical OH Activation of 1,3(4)-Diols. Angew. Chem. Int. Ed. 2020, 59, 19851 -19856. (i) Jeon, J. W.; He, Y.-T.; Shin, S.; Hong, S. Visible-Light-Induced ortho-Selective Migration on Pyridyl Ring: Trifluoromethylative Pyridylation of Unactivated Alkenes. Angew. Chem., Int. Ed. 2020, 59, 281-285. (j) Kong, W. Q.; Merino, E.; Nevado, C. Arylphosphonylation and Arylazidation of Activated Alkenes. Angew. Chem. Int. Ed. 2014, 53, 5078 -5082.

(12) For the migration of functional group with vacant electronic orbital, see: Jana, K.; Bhunia, A.; Studer, A. Radical 1,3Difunctionalization of Allylboronic Esters with Concomitant 1,2Boron Shift. Chem. 2020, 6, 512-522.

(13) For phosphite electrocyclic rearrangement, see: (a) Bentrude, W. G.; Lee, S. G.; Akutagawa, K.; Ye, W. Z.; Charbonnel, Y. Photoinduced electrocyclic rearrangements of allyl phosphites via possible phosphoranyl 1,3-biradicals. J. Am. Chem. Soc. 1987, 109, 1577-1579. For phosphite radical cation rearrangement, see: (b) Ganapathy, S.; Dockery, K. P.; Sopchik, A. E.; Bentrude, W. G. Photoinduced single electron transfer initiated rearrangements of 2phenylallyl phosphites. J. Am. Chem. Soc. 1993, 115, 8863-8864.

(14) (a) Smart, B. E. Fluorine Substituent Effects (on Bioactivity). J. Fluorine Chem. 2001, 109, 3-11. (b) Purser, S.; Moore, P. R.; Swallowb, S.; Gouverneur, V. Fluorine in medicinal chemistry. Chem. Soc. Rev. 2008, 37, 320-330. (c) Prchalová, E.; Štěpánek, O.; Smrček, S.; Kotora, M. Medicinal applications of perfluoroalkylated chaincontaining compounds. Future Med. Chem. 2014, 6, 1201-1229.

(15) (a) Romanenko, V. D.; Kukhar, V. P. Fluorinated Phosphonates: Synthesis and Biomedical Application. Chem. Rev. 2006, 106, 3868-3935. (b) Zhang, C. W; Li, Z. D; Zhu, L.; Yu, L. M Wang, Z. T; Li, C. Z. Silver-Catalyzed Radical Phosphonofluorination of Unactivated Alkenes. J. Am. Chem. Soc. 2013, 135, 14082-14085.

(16) Griller, D.; Ingold, K. U. Free-radical clocks. Acc. Chem. Res. 1980, 13, 317-323.

(17) Dolbier, W. R. Structure, Reactivity, and Chemistry of Fluoroalkyl Radicals. Chem. Rev. 1996, 96, 1557-1584.

(18) (a) Zhao, L.; Huang, Y.; Wang, Z.; Zhu, E.; Mao, T.; Jia, J.; Gu, J.; Li, X.-F.; He, C.-Y. Organophosphine-Catalyzed Difluoroalkylation of Alkenes. Org. Lett. 2019, 21, 6705-6709. (b) Helmecke, L.; Spittler, M.; Baumgarten, K.; Czekelius, C. Metal-Free Activation of C-I Bonds and Perfluoroalkylation of Alkenes with Visible Light Using Phosphine Catalysts. Org. Lett. 2019, 21, 782-7827. (c) Lu, H.; Wang, D.Y.; Zhang, A. Visible Light-Promoted Phosphine-Catalyzed Difluoroalkylation of Arenes and Heterocycles. J. Org. Chem. 2020, 85, 942-951.

(19) Wayner, D. D. M.; Houmam, A. Redox Properties of Free Radicals. Acta Chemica Scandinavica. 1998, 52, 377-384.

(20) For EPR studies for $\mathrm{P}^{\mathrm{V}}$ radical, see: Yoshimi Sueishi, Y.; NishiharaSpin, Y. Trapping of the Phosphorus-centered Radicals Generated from Hydrogen Abstraction Reaction by 2,2-Diphenyl-1picrylhydrazyl. Chem. Res. Chinese Universities. 2000, 16, 313-319. 


\section{Table of contents}

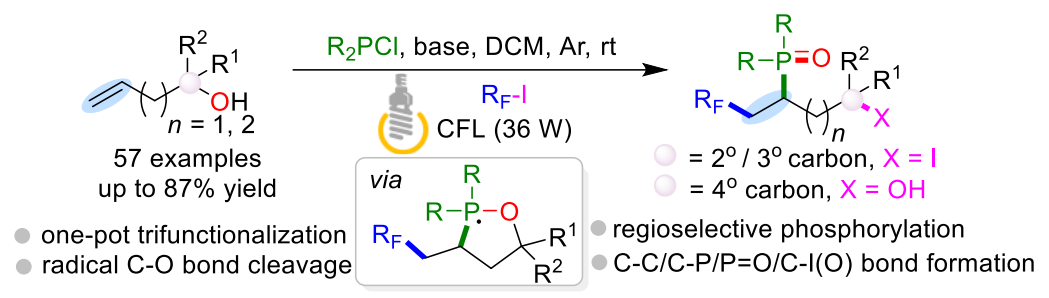

